http://dx.doi.org/ 10.22319/rmcp.v9i4.4651

Nota de investigación

\title{
Niveles de fumonisinas en rastrojo de maíz para consumo equino en el estado de Jalisco
}

\section{Levels of fumonisins in corn stover for equine consumption in Jalisco} State

Waldina Patricia Reyes-Velázquez ${ }^{\text {a* }}$

Claudia Nayeli Anguiano-Sevilla ${ }^{\mathrm{b}}$

Rubén Anguiano-Estrella ${ }^{\mathrm{b}}$

Luis Alfonso Jiménez-Ortega ${ }^{c}$

Pablo Torres-Morán ${ }^{\mathrm{d}}$

Federico Rojo ${ }^{\mathrm{e}}$

${ }^{a}$ Universidad de Guadalajara. Departamento de Salud Pública, Centro Universitario de Ciencias Biológicas y Agropecuarias (CUCBA). México.

${ }^{\mathrm{b}}$ Universidad de Guadalajara. Departamento de Producción Animal, CUCBA, México.

${ }^{\mathrm{c}}$ Universidad de Guadalajara. Licenciatura Ciencia de los Alimentos, CUCBA, México.

${ }^{\text {d } U n i v e r s i d a d ~ d e ~ G u a d a l a j a r a . ~ D e p a r t a m e n t o ~ d e ~ D e s a r r o l l o ~ R u r a l ~ S u s t e n t a b l e, ~ C U C B A . ~}$ México.

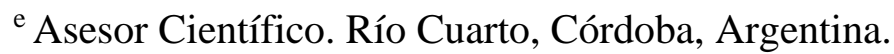

*Autor de correspondencia: waldinareyes2@gmail.com 


\section{- Resumen:}

A partir de casos confirmados de leucoencefalomalacia equina en el municipio de Juchitlán (estado de Jalisco), esta investigación tuvo como objetivo determinar la presencia y niveles de fumonisinas en el alimento. El área de estudio incluyó a los principales municipios en donde se concentra la población equina de Jalisco, teniendo en común el uso de prácticas similares en el manejo de la alimentación y conservación del alimento. Las determinaciones analíticas se realizaron mediante inmunoensayo de tipo competitivo y cromatografía de líquidos de alta resolución. Se detectó contaminación con fumonisinas en el $100 \%$ de muestras analizadas de todos los municipios, los niveles fueron de 0.23 a $19.18 \mathrm{mg} / \mathrm{kg}$. Se observó diferencia entre municipios $(P<0.001)$; la mayor concentración se observó en Ahualulco de Mercado (media: $12.83 \mathrm{mg} / \mathrm{kg}$ ) y la menor en Concepción de Buenos Aires (media: $0.27 \mathrm{mg} / \mathrm{kg}$ ). Del total de muestras analizadas, $62 \%$ superaron el límite máximo recomendado para equinos.

- Palabras clave: Fumonisinas, Rastrojo de maíz, Equinos.

\section{- Abstract:}

The objetive was to determine the presence and levels of fumonisins in feed from confirmed cases of equine leukoencephalomalacia from the Municipality of Juchitlán (Jalisco State). The field study included the 13 main municipalities where one of the biggest equine population in Jalisco is concentrated. They have a similar feeding management practices and feed preservation. The analytical determinations were performed by competitive type immunoassay and high-resolution liquid chromatography. Contamination with fumonisins was detected in $100 \%$ of analyzed samples; levels were from 0.23 to $19.18 \mathrm{mg} / \mathrm{kg}$. There was a difference between localities $(P<0.001)$; the highest concentration was observed in Ahualulco de Mercado (mean: $12.83 \mathrm{mg} / \mathrm{kg}$ ) and the lowest concentration in Concepción de Buenos Aires (mean: $0.27 \mathrm{mg} / \mathrm{kg}$ ). Of the total samples analyzed, $62 \%$ exceeded the recommended limit for horses.

- Key words: Fumonisins, Corn stover, Equine.

Recibido 02/10/2017

Aceptado 02/02/2018 
Las fumonisinas son metabolitos secundarios producidos por hongos filamentosos principalmente de las especies Fusarium verticillioides y $F$. proliferatum. Estas micotoxinas fueron caracterizadas en 1988 como diésteres de ácido tricarboxílico y aminopentol de cadena larga ${ }^{(1)}$. La contaminación natural de fumonisinas en alimentos se reporta con frecuencia en cereales como maíz y productos derivados para consumo humano y animal ${ }^{(2)}$. Condiciones ambientales estresantes favorecen la síntesis de fumonisinas, entre las cuales se describen sequía y temperaturas superiores a $30{ }^{\circ} \mathrm{C}$ durante el crecimiento de la planta, así como el almacenamiento inadecuado ${ }^{(3)}$.

Entre las fumonisinas descritas destacan la $\mathrm{FB}_{1}$ y $\mathrm{FB}_{2}$ por los efectos tóxicos en humanos y animales. Estudios epidemiológicos asocian la $\mathrm{FB}_{1}$ con el desarrollo de cáncer de esófago en humanos $^{(4)}$. El Centro de Investigaciones contra el Cáncer las clasifica como agentes probablemente cancerígenos dentro del grupo $2 \mathrm{~B}^{(5)}$. En equinos el consumo de alimentos contaminados con fumonisinas ocasiona el síndrome de leucoencefalomalacia equina (LEME), trastorno caracterizado por signos neurotóxicos como ceguera, ataxia, delirio, postración y convulsiones antes de morir ${ }^{(6)}$. El examen post-mortem muestra lesiones en encéfalo y tallo cerebral; además el estudio histopatológico permite observar pérdida de la arquitectura cerebral y necrosis de la materia blanca ${ }^{(7)}$.

A nivel mundial se han reportado numerosos brotes de LEME, con tasas de mortalidad del $85 \%{ }^{(7-10)}$. En el estado de Jalisco se presentan brotes de LEME con alta frecuencia, ocasionando grandes pérdidas económicas y afectivas; sin embargo, esta información no ha sido publicada en revistas científicas. En México, desde el año $1998^{(9)}$ a la fecha no existen datos públicos que permitan conocer la magnitud de este trastorno.

La Agencia de Administración de Alimentos y Medicamento de los Estados Unidos proporciona una guía sobre los niveles máximos recomendados de fumonisinas en alimentos de humanos y animales. En equinos se considera como límite máximo recomendado $5 \mathrm{mg} / \mathrm{kg}$ (ppm) en no más del $20 \%$ de la dieta ${ }^{(11)}$. En México el rastrojo de maíz es un subproducto destinado principalmente a la alimentación de rumiantes, sin embargo, frente a la escases de forrajes utilizados en la alimentación de equinos, como henos derivados de leguminosas (alfalfa) o de granos (avena), en algunas regiones del estado de Jalisco se destina el rastrojo de maíz como fuente de fibra en alto porcentaje de inclusión. Un estudio previo en la zona de los Altos mostró contaminación con $\mathrm{FB}_{1}$ en rastrojo de maíz blanco y amarillo, donde el $68 \%$ de las muestras analizadas fueron positivas con nivel máximo de $1.96 \mathrm{mg} / \mathrm{kg}^{(12)}$.

Este estudio fue llevado a cabo en el año 2012 cuando una serie de casos confirmados de LEME fueron divulgados en el ámbito local por médicos veterinarios del municipio de Juchitlán. En base a esta información, el objetivo del trabajo fue determinar la presencia y niveles de contaminación con fumonisinas en el ingrediente principal de la dieta. El estudio se extendió hacia los principales municipios donde se concentra la población equina del estado de Jalisco. 
Reseña de los casos. Tres (3) equinos machos pertenecientes a las razas Español (473 y 485 $\mathrm{kg}$ de peso corporal) y Criollo (464 $\mathrm{kg}$ de peso corporal) con edades de 3, 7 y 4 años, respectivamente se diagnosticaron por presentar LEME. Los animales recibían un promedio de $9 \mathrm{~kg}$ de ración por día con un ingesta mínima de rastrojo de maíz de $3.6 \mathrm{~kg} /$ día (40 \% de inclusión). El cuadro clínico observado en los animales incluyó ataxia, ceguera, sudoración profusa, hiperexcitabilidad y convulsiones antes de morir (en un lapso de $48 \mathrm{~h}$ ). Los estudios clínicos incluyeron examinación neurológica, análisis de biometría hemática y líquido cefalorraquídeo, todos dentro de los valores normales. Otras causas como traumas y mieloencefalitis equina protozoaria (MEP) se descartaron mediante estudios de rayos $\mathrm{X}$ y la técnica de Western Blot, resultando negativos en todos los casos. Por otra parte, en los mismos ranchos evaluados se detectó la presencia de otros caballos que comenzaron con síntomas leves aunque dentro del mismo cuadro. A diferencia, estos animales recibieron cuidados especiales con una dieta diferente, logrando lentamente su recuperación.

Recolección de muestras de alimento y municipios evaluados. Se obtuvieron 39 muestras de rastrojo de maíz (tres muestras de $500 \mathrm{~g} /$ municipio) a partir de los comederos en los ranchos ubicados en los municipios de Ahualulco de Mercado, Ameca, Autlán de Navarro, Concepción de Buenos Aires, Chiquilistlán, Cocula, Cuautitlán, El Grullo, Juchitlán, San Agustín, Tamazula de Gordiano, Tlajomulco de Zuñiga y Unión de Tula del Estado de Jalisco. Todas las muestras se mantuvieron en almacenamiento a $13 \%$ humedad y se molieron a un tamaño de partícula de $1 \mathrm{~mm}$ para su conservación en frascos de polietileno. Las muestras se analizaron por duplicado mediante la técnica de inmunoensayo enzimático de tipo competitivo (RomerLabs), y las muestras positivas que superaron el límite recomendado para equinos ${ }^{(11)}$, se confirmaron mediante la técnica de cromatografía de líquidos de alta resolución (HPLC). Las determinaciones analíticas se efectuaron en el área de micotoxicología del laboratorio de Residuos Tóxicos II, Departamento de Salud Pública, CUCBA, Universidad de Guadalajara. A partir de las muestras recolectadas se obtuvo una submuestra representativa por cuarteo para obtener dos muestras analíticas de $20 \mathrm{~g}$, las cuales se mezclaron con $100 \mathrm{ml}$ de metanol al 70\% durante 3 min en licuadora (Oster Modelo 4655013). Los extractos se filtraron en papel Whatman No.1 y se ajustó el pH a un rango entre 6 y 8; posteriormente se diluyó cada extracto en una relación 1:20 con agua desionizada.

El inmunoensayo (ELISA) se desarrolló conforme al protocolo descrito por el fabricante. Inicialmente, se realizó la mezcla del conjugado con los estándares de fumonisinas $(0,0.25$, $0.5,1.0,2.5$ y $5.0 \mathrm{ppm})$ o extractos de las muestras. Luego de transferir a los pocillos recubiertos con anticuerpos, la incubación se realizó durante $15 \mathrm{~min}$. Posteriormente se descartó su contenido y se procedió a lavar en forma repetida. La incubación con el sustrato enzimático se realizó durante $5 \mathrm{~min}$. Al detener la reacción, la cuantificación se realizó inmediatamente por lectura de la absorbancia a $450 \mathrm{~nm}$ con un filtro de referencia a $630 \mathrm{~nm}$ empleando un equipo STAT FAX 3200 (Technology Inc). El desempeño de inmunoensayo fue satisfactorio acorde a las características indicadas por el fabricante (coeficiente de 
regresión > 0.99). Durante la puesta a punto de la metodología, se comparó un mismo set de muestras positivas que fueron analizadas por HPLC (high performance liquid chromatography). Los resultados indicaron una fuerte correlación entre técnicas, siendo las muestras positivas por ELISA ratificadas por los resultados del HPLC.

Respecto al HPLC, la confirmación de las muestras positivas se realizó mediante la metodología propuesta por Shephard et $a l^{(13)}$ modificada por Doko et al ${ }^{(14)}$. Una alícuota de $50 \mu 1$ del extracto se derivatizó con $200 \mu 1$ de una solución de optaldialdehído (OPA) y $20 \mu 1$ fueron inyectados al sistema. La solución derivatizante se preparó disolviendo $40 \mathrm{mg}$ de OPA en $1 \mathrm{ml}$ de metanol, $5 \mathrm{ml}$ de tetraborato de $\mathrm{Na} 0.1 \mathrm{M}$ y $50 \mu \mathrm{l}$ de 2-mercaptoetanol. Las fumonisinas derivatizadas se analizaron empleando un sistema de detección de fluorescencia/HPLC fase reversa. El equipo HPLC consiste de un sistema isocrático con detector de fluorescencia Agilent 1100 (Palo Alto, CA, USA) y PC marca HP con el software ChemStation (Revisión A.10.01). Se empleó una columna C18 (150 x 4.6 mm, $5 \mu \mathrm{m}$ de tamaño de partícula; Supelcosil LC-ABZ, Supelco) conectada a una pre-columna Supelguard LC-ABZ. Como fase móvil se utilizó metanol-fosfato de sodio dihidrogenado 0.1 M (75:25, pH 3.35 ajustado con ácido ortofosfórico) a flujo de $1.5 \mathrm{ml} / \mathrm{min}$. Las longitudes de excitación y emisión fueron 335 y $440 \mathrm{~nm}$, respectivamente. La cuantificación se realizó midiendo la altura de los picos comparados con la curva de calibración a partir de soluciones testigo de $\mathrm{FB}_{1}$ y $\mathrm{FB}_{2}$ (SIGMA).

Para llevar a cabo el análisis estadístico, los niveles de fumonisinas en el alimento en cada muestra de rastrojo y procedente de cada municipio se transformaron logarítmicamente debido a que los mismos presentaron heterocedasticidad. La comparación de medias se realizó mediante la prueba de LSD de Fisher a un nivel de significancia de 0.001 utilizando el programa Minitab v. 14.0.

En relación a las condiciones ambientales, durante el desarrollo de este estudio se obtuvieron registros de temperatura y lluvias durante los años 2011 (siembra y cosecha del maíz) y 2012 (acopio). La consulta de los mismos se realizó mediante el acceso al portal de datos del Servicio Meteorológico Nacional ${ }^{(15)}$.

Durante el estudio se detectó que el $100 \%$ de los ranchos evaluados utilizaban el rastrojo de maíz en una proporción entre 40 y $50 \%$ de la dieta. Los demás ingredientes utilizados fueron en promedio maíz rolado (10 \%) y concentrados a base de cereales $(40-50 \%)$. El total de muestras analizadas presentó niveles detectables de fumonisinas $\left(\mathrm{FB}_{1}+\mathrm{FB}_{2}\right)$, los cuales fluctuaron entre 0.23 a $19.18 \mathrm{mg} / \mathrm{kg}$ (Cuadro 1). El análisis estadístico mostró diferencia significativa entre los municipios evaluados $(P<0.001)$, observándose la mayor concentración en Ahualulco de Mercado (media: $12.83 \mathrm{mg} / \mathrm{kg}$ ) y la menor en Concepción de Buenos Aires (media: $0.27 \mathrm{mg} / \mathrm{kg}$ ). El $62 \%$ de los municipios bajo estudio presentaron niveles de fumonisinas que superaron el valor recomendado. Las mayores concentraciones en el rastrojo y su relación con el valor máximo recomendado por la FDA $(5 \mathrm{mg} / \mathrm{kg})$ superó 
en algunos municipios dicho límite en diferente proporción, de mayor a menor se destaca: Ahualulco de Mercado (19.18 mg/kg; 3.8 veces), El Grullo (10.91 mg/kg; 2.2 veces), Tlajomulco de Zuñiga (6.32 mg/kg; 1.3 veces), Unión de Tula $(5.44 \mathrm{mg} / \mathrm{kg} ; 1.1$ veces), Autlán de Navarro y Cocula $(5.02 \mathrm{mg} / \mathrm{kg} \text {; en el límite del valor máximo recomendado })^{(11)}$.

Cuadro 1: Niveles de fumonisinas totales ( $\mathrm{mg} / \mathrm{kg}$ ) en el rastrojo de maíz obtenido de ranchos a partir de los principales municipios del estado de Jalisco en 2012

\begin{tabular}{|l|c|c|}
\hline Localidad & Media \pm DE & Rango \\
\hline Ahualulco de Mercado & $12.83 \pm 6.35 \mathrm{a}$ & $6.48-19.18$ \\
\hline Ameca & $1.55 \pm 0.46 \mathrm{~d}$ & $1.04-1.98$ \\
\hline Autlán de Navarro & $3.15 \pm 1.66 \mathrm{~cd}$ & $1.58-5.02$ \\
\hline Concepción de Buenos Aires & $0.27 \pm 0.04 \mathrm{f}$ & $0.23-0.30$ \\
\hline Chiquilistlán & $0.30 \pm 0.03 \mathrm{f}$ & $0.27-0.33$ \\
\hline Cocula & $4.68 \pm 0.35 \mathrm{abc}$ & $4.33-5.02$ \\
\hline Cuautitlán & $0.78 \pm 0.17 \mathrm{ef}$ & $0.60-0.95$ \\
\hline El Grullo & $7.90 \pm 2.22 \mathrm{ab}$ & $5.95-10.91$ \\
\hline Juchitlán & $0.36 \pm 0.02 \mathrm{f}$ & $0.34-0.38$ \\
\hline San Agustín & $2.97 \pm 0.32 \mathrm{bcd}$ & $2.65-3.29$ \\
\hline Tamazula de Gordiano & $1.29 \pm 0.91 \mathrm{ef}$ & $0.32-2.45$ \\
\hline Tlajomulco de Zuñiga & $3.71 \pm 2.61 \mathrm{~cd}$ & $0.35-6.32$ \\
\hline Unión de Tula & $3.69 \pm 1.44 \mathrm{bcd}$ & $2.00-5.44$ \\
\hline
\end{tabular}

$\mathrm{DE}=$ desviación estándar.

Las literales indican diferencia estadística entre localidades $(P<0.001)$.

Debido a la naturaleza endófita de las especies de Fusarium en maíz, la producción de fumonisinas puede ocurrir en etapas tempranas del cultivo y la maduración del grano. En el reporte de las condiciones climáticas durante 2011 se observa de forma general que existieron condiciones adecuadas de temperatura y humedad para el cultivo, y eventualmente también para el desarrollo de especies de Fusarium, entre ellas toxicogénicas. En los meses de mayo a noviembre de 2011, la temperatura promedio fue $22.1^{\circ} \mathrm{C}$ (rango de 14.7 a $29.5^{\circ} \mathrm{C}$ ) y las precipitaciones fluctuaron entre 0.2 y $203.8 \mathrm{~mm}$. En relación al año 2012, la temperatura promedio fue de $20.4{ }^{\circ} \mathrm{C}$ (rango de 12.7 a $28.1^{\circ} \mathrm{C}$ ) y la precipitación pluvial entre $0.1 \mathrm{a} 202.2 \mathrm{~mm}^{(15)}$.

Las investigaciones relacionadas con brotes en campo de LEME en caballos reportan variaciones importantes en los niveles detectados de fumonisinas en los alimentos asociados. Destacan los observados en algunas regiones de Estados Unidos de Norteamérica, durante 
1989 y 1990 se presentaron 45 casos de LEME asociados al consumo de alimentos elaborados principalmente con grano de maíz parcialmente molido. Las concentraciones de $\mathrm{FB}_{1}$ fueron de $<1$ a $126 \mu \mathrm{g} / \mathrm{g}$, la mayoría de las muestras con niveles superiores a $10 \mu \mathrm{g} / \mathrm{g}^{(8)}$. En Brasil se presentaron casos de LEME asociados a la detección de fumonisinas en niveles de 0.2 a $38.5 \mu \mathrm{g} / \mathrm{g}$, destaca la presencia del rastrojo de maíz como uno de los ingredientes en la alimentación de los caballos afectados ${ }^{(16)}$; mientras que en otro reporte observado en Sao Paulo, dos casos de LEME se asociaron con niveles de $\mathrm{FB}_{1}$ de 0.12 y $0.02 \mu \mathrm{g} / \mathrm{g}$ respectivamente $^{(7)}$. En México sólo se ha publicado un brote de LEME que ocasionó la muerte de 100 burros en el estado de Oaxaca, 14 muestras del alimento consumido presentaron niveles de fumonisinas de 0.67 a $13.3 \mu \mathrm{g} / \mathrm{g}^{(9)}$. En Argentina, Brasil y Uruguay se han diagnosticado casos de LEME en caballos y mulas asociados con el consumo de maíz (mezclas de granos, mazorcas, pajas, etc.) además de subproductos contaminados. Por otra parte, en otro estudio realizado en Brasil, se encontró que los brotes de LEME estaban asociados al consumo de $1 \mathrm{~kg}$ de maíz por día o dietas conteniendo al menos $20 \%$ de maíz; en animales de diferentes edades con morbilidad de 4 a $100 \%$ y mortalidad cercana al $100 \%{ }^{(17,18)}$.

En este estudio, los niveles de contaminación por fumonisinas que se reportan se obtuvieron del principal ingrediente encontrado en la dieta (rastrojo de maíz). Se considera que existe la posibilidad de que los animales en todos los ranchos hayan estado expuestos a niveles fluctuantes de fumonisinas. Dado que las micotoxinas exhiben una distribución heterogénea en el alimento, la muestra analítica obtenida del comedero representa una evidencia de que existió exposición a fumonisinas. Por otra parte, las especies toxicogénicas de Fusarium infectan una diversidad de cereales que forman parte de los concentrados alimenticios. Por esta razón, otros ingredientes en la dieta podrían haber incrementado el nivel de exposición. En el municipio de Juchitlán, el hallazgo de fumonisinas directamente del rastrojo de maíz en el comedero (media $0.36 \pm 0.02 \mathrm{mg} / \mathrm{kg}$ ) junto con los signos clínicos neurológicos e histopatológicos se confirma el diagnóstico de LEME. Estos resultados de fumonisinas se encuentran dentro del rango de valores informados en otros estudios ${ }^{(7,8,16)}$.

En relación a los niveles de fumonisinas en rastrojo de maíz detectado en los 13 municipios, los resultados fueron similares a los reportados previamente en alimentos de equinos. Entre estos, destacan los realizados en Rio de Janeiro (Brasil) en cinco ranchos de caballos donde los niveles de fumonisinas detectados fueron de 0.1 a $7.49 \mu \mathrm{g} / \mathrm{g}$, ninguno de los niveles promedio excedió el límite recomendado ${ }^{(19)}$. En Alemania se detectó FB $_{1}$ en concentrados, peletizados, maíz, avena y cebada; los resultados mostraron contaminación en 94 \% de las muestras analizadas, los niveles fueron de 2 a $2200 \mu \mathrm{g} / \mathrm{kg}$, y las mayores concentraciones se observaron en muestras de maíz y alimento peletizado ${ }^{(20)}$.

La temperatura y estrés hídrico son los principales factores que influyen en el desarrollo de especies de Fusarium; sin embargo, el impacto de estos factores climáticos depende de otras 
variables, tales como infecciones fúngicas adicionales, sequía o factores del huésped ${ }^{(3)}$. Durante el desarrollo del maíz, dos períodos resultan críticos en la contaminación de los granos con fumonisinas, uno de ellos es la floración y otro el secado de los granos durante la maduración de la mazorca. Los estudios identifican que baja disponibilidad de agua $\left(\mathrm{a}_{\mathrm{w}}\right)$ y elevadas temperaturas están asociadas a la presencia de fumonisinas durante la floración. En cambio, lluvias o altas temperaturas pueden desencadenar también la síntesis de fumonisinas durante la cosecha ${ }^{(21)}$.

La temperatura óptima para el crecimiento de $F$. verticillioides es de $25{ }^{\circ} \mathrm{C}$ con marcada reducción a los $15{ }^{\circ} \mathrm{C}$ o por efecto de la $\mathrm{a}_{\mathrm{w}}$. Se ha demostrado que la disminución del $\mathrm{a}_{\mathrm{w}}$ en $F$. verticillioides incrementa la expresión del gen $F U M 1$ que codifica para la síntesis de fumonisinas ${ }^{(21,22)}$. No obstante, esta asociación no es directa, ya que existen evidencias de que la producción de fumonisinas ocurre bajo diversas condiciones. En Uganda, elevada humedad y prácticas agrícolas inadecuadas se asociaron con mayores niveles de fumonisinas $^{(23)}$. Según los datos recolectados por esta investigación, los registros de temperatura y precipitaciones en el estado de Jalisco durante 2011 y 2012 resultaron potencialmente favorables para la ocurrencia y proliferación de especies de Fusarium productoras de fumonisinas.

Se concluye que el rastrojo de maíz destinado al consumo de equinos presentó niveles de fumonisinas asociado a casos de LEME confirmados durante 2012. Las concentraciones presentes en los diferentes municipios del estado de Jalisco permitieron identificar a Ahualulco de Mercado, Autlán de Navarro, Cocula, El Grullo, Tlajomulco de Zuñiga y Unión de Tula como zonas de riesgo de presentación de casos de LEME.

\section{- Literatura citada:}

1. Gelderbrom WC, Marasas WFO, Vleggar R, Thiel PG, Cawood ME. Fumonisins: isolation, chemical characterisation and biological effects. Mycopathologia 1992;(117):11-16.

2. Deepa N, Sreenivasa MY. Fumonisins: A review on its global occurrence, epidemiology, toxicity and detection. J Vet Med Res 2017;4(6):1093-1102.

3. Marin P, Magan N, Vázquez C, González-Jaén MT. Differential effect of environmental conditions on the growth and regulation of the fumonisin biosythetic gene FUMI in the maize producer Fusarium verticillioides and Fusarium proliferatum. Microbiol Ecol 2010;(73):303-311. 
4. Alizadeh AM, Roshamdel G, Roudbarmohammadi S, Roudbary M, Sohanaki H, Ghiasian SA, et al. Fumonisin B1 contamination of cereals and risk of esophageal cancer in a high risk area in northeastern Iran. Asian Pacific J Cancer Prev 2012;(13):26252628.

5. IARC. International Agency for Research on Cancer. Some traditional herbal medicines, some mycotoxins, naphthalene and styrene. Monographs on the evaluation of carcinogenic risks to humans. Lyon 2002;(82):33.

6. Caloni F, Cortinovis C. Effects of fusariotoxins in the equine species. Vet $\mathrm{J}$ 2010;(186):157-161.

7. Vendruscolo CP, Frias NC, de Carvalho CB, de Sá LMR, Belli CB, Baccarin RYA. Leukoencephalomalacia outbreak in horses due to consumption of contaminated hay. J Vet Intern Med 2016;(30):1879-1881.

8. Ross PF, Rice LG, Reagor JC, Osweiler GD, Wilson TM, Nelson HA, et al. Fumonisin B1 concentrations in feeds from 45 confirmed equine leukoencephalomalacia cases. $\mathrm{J}$ Vet Diagn Invest 1991;(3):238-241.

9. Rosiles MR, Bautista J, Fuentes VO, Ross F. An outbreak of equine leukoencephalomalacia at Oaxaca, Mexico, associated with fumonisin B1. J Vet Med Series A 1998;45(5):299-302.

10. Giannitti F, Sain SD, Pacin AM, Barrandeguy M, Larrere C, Ortega J, Uzal FA. Equine leukoencephalomalacia (ELEM) due to fumonisins B1 and B2 in Argentina. Pesq Vet Bras 2011;31(5):407-412.

11. FDA, Food and Drug Administration, Center for Food and Applied Nutrition. Guidance for Industry. Fumonisin levels in human foods and animal feeds 2001. https://www.fda.gov/Food/GuidanceRegulation/GuidanceDocumentsRegulatoryInfor mation/ucm109231.htm

12. Arias S, Patricio SM, Theumer M, Rubinstein H, Reyes-Velázquez WP. Ocurrencia natural de fumonisinas en rastrojo de maíz procedente de diferentes variedades e híbridos en el estado de Jalisco [resumen]. Semana de la Investigación Científica, CUCBA. Universidad de Guadalajara 2010:146.

13. Shephard GC, Sydenham EW, Thiel PG, Gelderblom WCA. Quantitative determination of fumonisins B1 and B2 by high-performance liquid chromatography with fluorescence detection. J Liq Chromatogr 1990;(13):2077-2080. 
14. Doko B, Rapior S, Visconti A, Schjoth J. Incidence and levels of fumonisins contamination in maize genotypes grown in Europe and Africa. J Agric Food Chem 1995;(43):429-434.

15. CONAGUA. Comisión Nacional del Agua. Servicio Meteorológico Nacional. Reporte Anual 2011 y 2012.

16. Sydenham EW, Marasas WFO, Shephard GS, Thiel PG, Hirooka EY. Fumonisin concentrations in Brazilian feeds associated with field outbreaks of confirmed and suspected animal mycotoxicosis. J Agric Food Chem 1992;(40):994-997.

17. Riet-Correa F, Rivero R, Odriozola E, Adrien ML, Medeiros RMT, Schild AL. Mycotoxicoses of ruminants and horses. J Vet Diagn Invest 2013;25(6):692-708.

18. Rivero R, Collazo S, Ugarte E, Nan F, Mazzolini Y, Matto C. Leukoencephalomalacia in equine on the West Coast of Uruguay. Veterinaria (Montevideo) 2013;49(189):3139.

19. Keller KM, Queiroz BD, Keller LAM, Ribeiro JMM, Cavaglieri LR, González ML, et $a l$. The mycobiota and toxicity of equine feeds. Vet Res Commun 2007;(31):1037-1045.

20. Liessener K, Curtui V, Bletrich R, Märtinbauer E, Usleber E. Mycotoxins in horse feed. Mycotoxin Res 2010;(23):26-30.

21. Santiago R, Cao A, Butron A. Genetic factors involved in fumonisin accumulation in maize kernels and their implication in maize agronomic management and breeding. Toxins 2015;7(8):3267-3296.

22. Jurado M, Marín P, Magan N, Gonzalez-Jaén MT. Relationship between solute and matric potential stress, temperature, growth and FUM1 gene expression in two Fusarium verticillioides strains from Spain. Applied and Environm Microbiol 2008;74(7):20322036.

23. Atukwase A, Kaaya AN, Muyanja C. Factors associated with fumonisin contamination of maize in Uganda. J Sci Food Agric 2009;(89):2393-2398. 